\title{
Role of Phenotypes of Circulating Tumor Cells in the Diagnosis and Treatment of Colorectal Cancer
}

\author{
Yuchen Zhong $\mathbb{D}^{1,2}$ \\ Tianyi Ma $\mathbb{D}^{\prime}$ \\ Tianyu Qiao' \\ Hanqing $\mathrm{Hu}^{\prime}$ \\ Zhengliang $\mathrm{Li}^{\prime}$ \\ Kangjia Luo' \\ Yuliuming Wang \\ Qingchao Tang $\mathbb{D}^{\prime}$ \\ Guiyu Wang (D) ${ }^{1,2}$ \\ Rui Huang' \\ Xishan Wang ${ }^{3}$
}

'Department of Colorectal Surgery, The Second Affiliated Hospital of Harbin

Medical University, Harbin, Heilongjiang, I50080, People's Republic of China;

${ }^{2}$ Department of Colorectal Surgery, The Cancer Hospital of the University of

Chinese Academy of Sciences (Zhejiang

Cancer Hospital), Hangzhou, Zhejiang,

310002, People's Republic of China;

${ }^{3}$ Department of Colorectal Surgery,

National Cancer Center/Cancer

Hospital, Chinese Academy of Medical

Science and Peking Union Medical

College, National Clinical Research

Center for Cancer, Beijing, I0002I,

People's Republic of China
Correspondence: Guiyu Wang; Rui Huang Department of Colorectal Surgery, The Second Affiliated Hospital of Harbin Medical University, Harbin, Heilongjiang, 150080, People's Republic of China $\mathrm{Tel} / \mathrm{Fax}+86-19558225177$;

+86-15204620800

Email guiyWang@163.com;

huangrui2019@I63.com
Objective: To investigate the relationship between circulating tumor cells (CTCs) and their subpopulations and colorectal cancer (CRC). To explore the application of CTCs' numbers and positive rates in the diagnosis and treatment of $\mathrm{CRC}$, and to assess the effect of surgery on CTCs numbers and positivity.

Methods: We identified CTCs using the CanPatrol technique after enrollment. Peripheral blood samples were collected from 74 CRC patients before anti-tumor treatment. CTCs can be divided into the following three phenotypes: epithelial CTCs (E-CTCs) (EpCAM+, Vimentin-), mesenchymal CTCs (M-CTCs) (EpCAM-, Vimentin+), and mixed CTCs (E/ M-CTCs) (EpCAM+, Vimentin+). CTCs and the proportion of subtypes were statistically compared with clinicopathological characteristics.

Results: The positive rate of M-CTCs was significantly higher in patients with tumor size $\geq 5 \mathrm{~cm}(85.7 \%$ vs $49.1 \%, \mathrm{P}=0.004)$ and carcinoembryonic antigen $(\mathrm{CEA})>5 \mathrm{ng} / \mathrm{mL}(83.3 \%$ vs $51.0 \% \mathrm{p}=0.024)$. Moreover, the T stage (T1 0, T2 33.3\%, T3 59.4\%, T4 100\%, p < 0.0005 ) and TNM stage (stage I $11.8 \%$, stage II $79.2 \%$, stage III $64.3 \%$, stage IV $100 \%$, p < $0.0005)$ were correlated with the positive rate of M-CTCs. We also found that the proportion of M-CTCs was correlated with the T stage $(\mathrm{p}<0.0005)$ and TNM stage $(\mathrm{p}=0.0200)$, but not with the $\mathrm{N}$ stage $(\mathrm{p}=0.6889)$. In survival analysis, M-CTCs $>1$ were found associated with worse disease-free survival $(\mathrm{p}=0.007)$. After treatment, the number and proportion of CTCs and M-CTCs were significantly reduced.

Conclusion: The positive rate of M-CTCs was associated with tumor size, T stage, TNM stage, vascular invasion, and CEA. As the disease progressed, the proportion of M-CTCs gradually increased, and the survival performance was worse in patients with a high positive rate of M-CTCs.

Keywords: circulating tumor cells, colorectal cancer, surgery, EMT

\section{Introduction}

Colorectal cancer (CRC) was the second most common cause of cancer-related deaths worldwide in 2018. The incidence of CRC in 2018 was $11 \%$ with a total of $1,800,977$ newly diagnosed cases, and it ranked third in the incidence of cancers. ${ }^{1}$ Diagnosis of CRC is mainly based on biopsy samples taken during endoscopy. Complete colonoscopy or CT colonography is mandatory to detect synchronous cancers that are present in about $2-4 \%$ of patients. ${ }^{2}$ Hematogenous metastasis is initiated by cancer cells that are transported through the circulation, also known as circulating tumor cells (CTCs), from the primary tumor to vital distant organs, 
and it is directly responsible for most cancer-related deaths. $^{3-5}$ As monitoring of hematogenous metastasis of the tumor is a great challenge in cancer patients prior to the novel blood tumor cell detection methods, the test of CTCs is emerging as a real-time "liquid biopsy" in cancer patients, which can reflect the progress and prognosis of the disease. In breast cancer and CRC, it has been proved that multiple measurements of CTCs can be used as follow-up indicators and for evaluating the treatment efficacy. $^{6-9}$

According to epithelial cell adhesion molecule and vimentin expression, CTCs can be divided into the epithelial phenotype (E-CTCs), mesenchymal cell phenotype
(M-CTCs), and mixed epithelial and mesenchymal cells' phenotype (E/M-CTCs) and circulating tumor microemboli (CTMs). ${ }^{10,11}$ As the epithelial-mesenchymal transformation (EMT) plays an important role in the biological progress of metastasis, some studies have shown that the EMT phenotype of CTCs may be related to early metastasis. ${ }^{12}$ EMT is partially responsible for cancer invasion, metastasis, and chemoresistance in CRC. Previous studies have shown that M-CTCs are closely associated with the prognosis of gastric, breast, and liver cancers. ${ }^{13-15}$ It is helpful to identify more aggressive tumor cells by EMT labeling of CTCs and to evaluate the prognosis and determine suitable therapies for aggressive CTCs. ${ }^{16}$
A
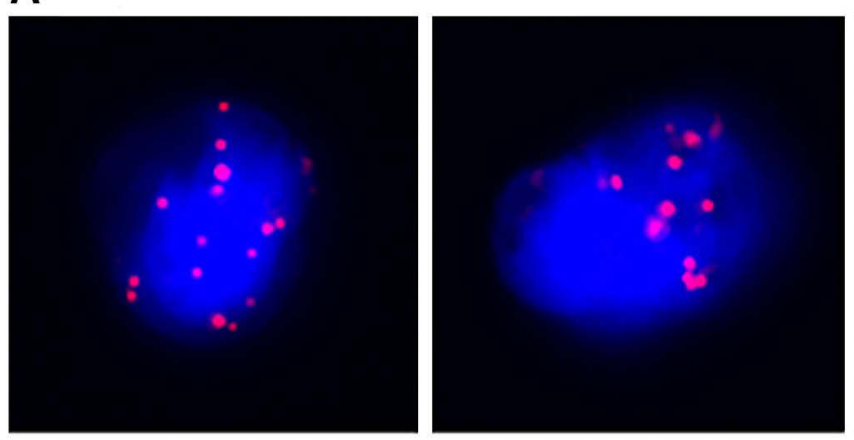

B
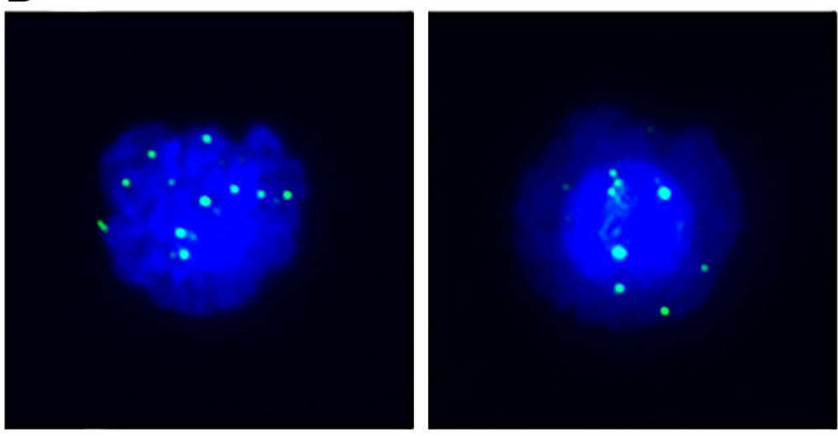

C
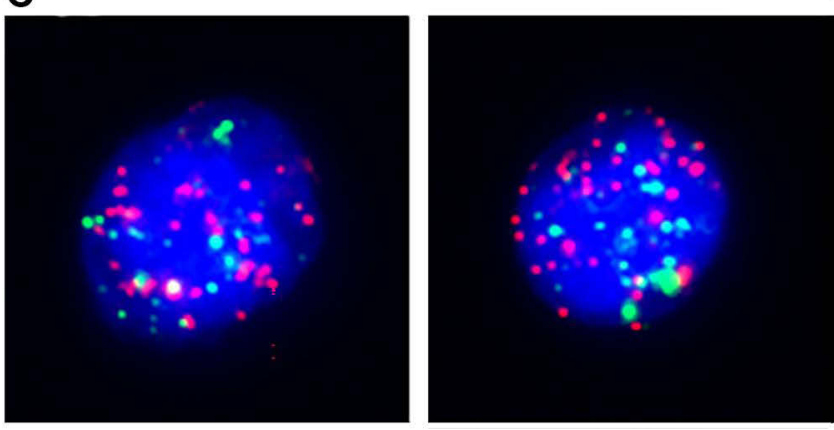
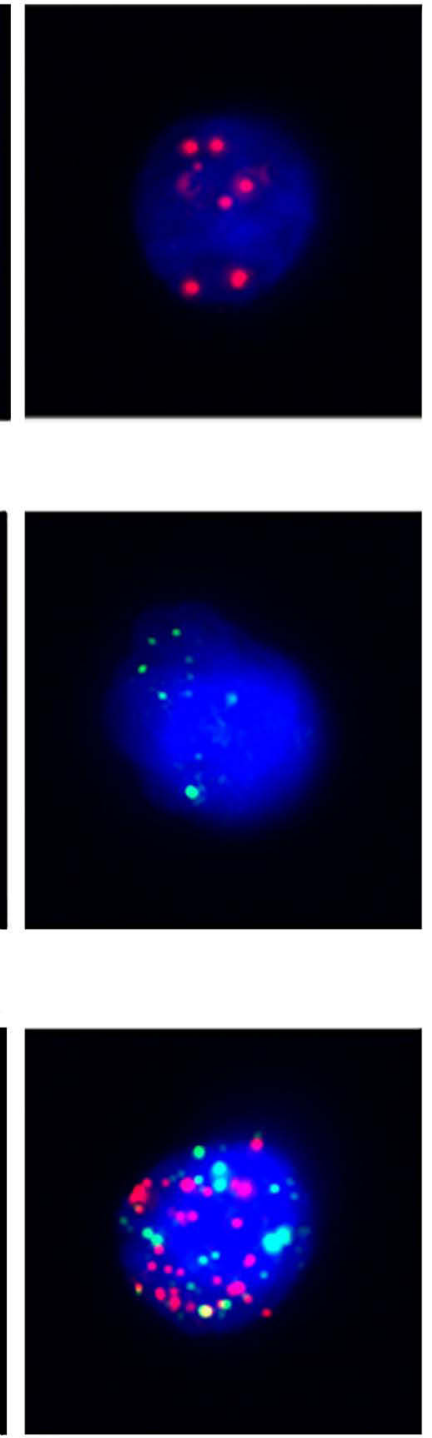

Figure I Representative images of the detection of CTCs in the peripheral blood of patients with colorectal cancer. (A) EpCAM expression (red fluorescence) in epithelial CTCs; (B) vimentin expression (green fluorescence) in mesenchymal CTCs; (C) EpCAM/vimentin expression (red and green fluorescence) in epithelial-mesenchymal CTCs. Abbreviation: CTCs, circulating tumor cells. 


\section{Methods}

\section{Patient Information and Research Ethics}

This was a prospective, open-label, cohort-controlled trial. Patients were admitted to our hospital from January 2019 to September 2020 with definite biopsy CRC pathology reports before the operations were selected. All patients completed the relevant preoperative examination.

The clinical characteristics, such as age, gender, American Society of Anesthesiologists category, body mass index (BMI), location of the tumor, smoking habit, and blood tumor markers, were collected. The pathology parameters, including histological differentiation and clinical stage according to the WHO TNM classifications 2017, were also collected. All patients signed the informed consent form according to the research ethics permitted by the research ethics committee of the Second Affiliated Hospital of Harbin Medical University and our study was conducted in accordance with the Helsinki Declaration.

\section{Inclusion and Exclusion Criteria}

Inclusion criteria were as follows: (1) Pathological diagnosis of colorectal adenocarcinoma. (2) Age from 18 to 80 years. (3) Eastern Cooperative Oncology Group (ECOG) physical strength score $<3$ points. (4) No treatment before surgery. (5) Patients signed the informed consent form.

Exclusion criteria were as follows: (1) Indications for emergency surgery, such as acute intestinal obstruction or perforation. (2) More than one primary tumor location. (3) Patient decided to quit the trial before the second CTCs test.

\section{Detection of CTCs}

CTCs detection time point: The first CTCs detection was performed before the beginning of surgery. The second examination was performed at the 8th week postoperatively, and all inflammatory markers were within the normal range, as described previously.

CTCs detection technology: The assessment of CTCs numbers and phenotype was based on the CanPatrol ${ }^{\circledR}$ Technique, detailed protocol has already been published. ${ }^{14,17}$ Specifically, the CanPatrol CTCs filtration system consists of a filter tube, a manifold vacuum plate with valve settings, an E-Z 96 vacuum manifold, and a vacuum pump. Red blood cells were removed by lysis
Table I Clinicopathological Characteristics of the Patients and Detection of CTCs

\begin{tabular}{|c|c|c|c|}
\hline Parameter & $\begin{array}{c}\text { Number of } \\
\text { CTCs }\end{array}$ & $\begin{array}{c}\text { Number of } \\
\text { Cases }\end{array}$ & $p$-value \\
\hline Age & & & 0.170 \\
\hline$\leq 60$ & $16.3 \pm 15.1$ & 28 & \\
\hline$>60$ & $24.4 \pm 18.8$ & 46 & \\
\hline Gender & & & 0.646 \\
\hline Male & $22.0 \pm 18.7$ & 48 & \\
\hline Female & $20.3 \pm 16.6$ & 26 & \\
\hline Location & & & 0.309 \\
\hline Rectum & $23.5 \pm 19.8$ & 38 & \\
\hline Left Hemicolon & $17.1 \pm 17.0$ & 19 & \\
\hline Right Hemicolon & $20.5 \pm 13.5$ & 16 & \\
\hline Size of the tumor & & & 0.362 \\
\hline$\leq 5 \mathrm{~cm}$ & $19.6 \pm 16.4$ & 53 & \\
\hline$>5 \mathrm{~cm}$ & $25.8 \pm 20.8$ & 21 & \\
\hline $\mathrm{CEA}^{\#}$ & & & $0.014 *$ \\
\hline$\leq 5 \mathrm{ng} / \mathrm{mL}$ & $17.5 \pm 15.1$ & 49 & \\
\hline$>5 \mathrm{ng} / \mathrm{mL}$ & $30.9 \pm 20.8$ & 18 & \\
\hline CA199\# & & & 0.538 \\
\hline$\leq 37 \mathrm{U} / \mathrm{mL}$ & $21.3 \pm 18.4$ & 58 & \\
\hline$>37 \mathrm{U} / \mathrm{mL}$ & $21.6 \pm 14.7$ & 9 & \\
\hline T stage & & & 0.213 \\
\hline TI & $9.3 \pm 6.6$ & 9 & \\
\hline $\mathrm{T} 2$ & $15.0 \pm 6.4$ & 12 & \\
\hline $\mathrm{T} 3$ & $21.1 \pm 17.6$ & 32 & \\
\hline $\mathrm{T} 4$ & $29.2 \pm 21.6$ & 21 & \\
\hline $\mathrm{N}$ stage & & & 0.334 \\
\hline No & $19.4 \pm 17.8$ & 42 & \\
\hline $\mathrm{NI/N2}$ & $24.0 \pm 17.9$ & 32 & \\
\hline TNM stage & & & 0.605 \\
\hline 1 & $12.8 \pm 7.4$ & 17 & \\
\hline ॥ & $24.1 \pm 21.3$ & 24 & \\
\hline III & $24.2 \pm 18.6$ & 28 & \\
\hline IV & $19.3 \pm 11.9$ & 5 & \\
\hline Differentiation & & & 0.909 \\
\hline Poor & $22.0 \pm 20.4$ & 13 & \\
\hline $\begin{array}{l}\text { Moderate and well } \\
\text { differentiated }\end{array}$ & $21.2 \pm 17.5$ & 61 & \\
\hline Vascular invasion & $22.6 \pm 19.1$ & - & 0.675 \\
\hline Lymphatic invasion & $25.6 \pm 22.8$ & - & 0.217 \\
\hline Nerve invasion & $20.7 \pm 18.0$ & - & 0.646 \\
\hline
\end{tabular}

Notes: ${ }^{p}<<0.05$, "lack of data.

Abbreviations: CTCs, circulating tumor cells; CEA, carcinoembryonic antigen; CA199, carbohydrate antigen 199; TNM, Tumor-Node-Metastasis. 
buffer before filtration. After that, the remaining cells were resuspended in phosphate-buffered saline (PBS) and 4\% formaldehyde. Then, interception of CTCs was performed by nanofiltration technology. To distinguish circulating tumor cells from leukocytes, CD45 was used as a leukocyte marker. The phenotypes of CTCs were classified into the following three subgroups using the RNAISH method: E-type (EpCAM expression and CD45-), M-type (Vimentin expression and CD45-), and E/M-type (EpCAM/vimentin expression and CD45-) (Figure 1).

\section{Statistical Methods}

SPSS 23.0 (IBM Corp., Armonk, NY, USA) and GraphPad Prism 8.0 (GraphPad Software Inc., La Jolla, CA, USA) were used for statistical analysis. Quantitative data were tested by the Student's $t$-test or a nonparametric test (Wilcoxon test or Mann-Whitney $U$-test) based on whether they conformed to normal distribution. Categorical variables were compared using the $\chi^{2}$ test or the Fisher's exact test.

\section{Results}

Seventy-four CRC patients met our criteria between January 2019 and September 2020. Table 1 summarizes the clinicopathological features of 74 CRC patients. E-CTCs were found in the peripheral blood of 33 patients (44.6\%), E/M-CTCs were found in the peripheral blood of 71 patients $(95.9 \%)$, and M-CTCs were found in the peripheral blood of 64 patients (86.5\%). CTMs were not detected. Analysis of the clinicopathological information revealed that the number of CTCs was generally higher in patients with carcinoembryonic antigen (CEA) levels exceeding normal values $(30.9 \pm 20.8$ vs $17.5 \pm 15.1$, $\mathrm{P}=0.014)$. Moreover, no statistical differences were found in the other parameters.

To further explore the relationship between CTCs and CRC, in this study, the number of M-CTCs $>1$ cell/ $7.5 \mathrm{~mL}$ peripheral blood was defined as positive. Table 2 shows that the positive rate of M-CTCs was statistically correlated with many indicators. M-CTCs positive patients were more likely to have a tumor size $\geq 5 \mathrm{~cm}$ than M-CTCs negative patients $(85.7 \%$ vs $49.1 \%, \mathrm{p}=0.004)$. The positive rate of CEA in M-CTCs positive patients was also significantly higher $(83.3 \%$ vs $51.0 \%, \mathrm{p}=0.024)$. Among the pathological indexes, the positive rate of M-CTCs
Table 2 Correlation Between Positive Rate of M-CTCs and Clinicopathological Characteristics

\begin{tabular}{|c|c|c|}
\hline & $\begin{array}{c}\text { Positive Rate of } \\
\text { M-CTCs }\end{array}$ & $p$-value \\
\hline All cases & $59.5 \%$ & \\
\hline Age & & 0.228 \\
\hline$\leq 60$ & $50.0 \%$ & \\
\hline$>60$ & $65.2 \%$ & \\
\hline Gender & & 1.000 \\
\hline Male & $60.4 \%$ & \\
\hline Female & $57.7 \%$ & \\
\hline Location & & 0.813 \\
\hline Rectum & $57.9 \%$ & \\
\hline Left Hemicolon & $57.9 \%$ & \\
\hline Right Hemicolon & $68.8 \%$ & \\
\hline Size of the tumor & & $0.004 * *$ \\
\hline$\leq 5 \mathrm{~cm}$ & $49.1 \%$ & \\
\hline$>5 \mathrm{~cm}$ & $85.7 \%$ & \\
\hline $\mathrm{CEA}^{\#}$ & & 0.024 \\
\hline$\leq 5 \mathrm{ng} / \mathrm{mL}$ & $51.0 \%$ & \\
\hline$>5 \mathrm{ng} / \mathrm{mL}$ & $83.3 \%$ & \\
\hline CAI99\# & & 0.471 \\
\hline$\leq 37 \mathrm{U} / \mathrm{mL}$ & $58.6 \%$ & \\
\hline$>37 \mathrm{U} / \mathrm{mL}$ & $66.7 \%$ & \\
\hline T stage & & $0.000 * *$ \\
\hline TI & 0 & \\
\hline $\mathrm{T} 2$ & $33.3 \%$ & \\
\hline $\mathrm{T} 3$ & $59.4 \%$ & \\
\hline T4 & $100 \%$ & \\
\hline$N$ stage & & 0.232 \\
\hline No & $52.4 \%$ & \\
\hline $\mathrm{NI/N2}$ & $68.8 \%$ & \\
\hline TNM stage & & $0.000 * *$ \\
\hline I & $11.8 \%$ & \\
\hline II & $79.2 \%$ & \\
\hline III & $64.3 \%$ & \\
\hline IV & $100 \%$ & \\
\hline Differentiation & & 0.356 \\
\hline Poor & $46.2 \%$ & \\
\hline Moderate and well & $62.3 \%$ & \\
\hline differentiated & & \\
\hline Vascular invasion & $80.0 \%$ & $0.023 *$ \\
\hline Lymphatic invasion & $69.2 \%$ & 0.208 \\
\hline Nerve invasion & $60.8 \%$ & 0.766 \\
\hline
\end{tabular}

Notes: $* p<0.05, * * p<0.005$, " lack of data.

Abbreviations: M-CTCs, mesenchymal circulating tumor cells; CEA, carcinoembryonic antigen; CA199, Carbohydrate antigen 199; TNM, Tumor-Node-Metastasis. 

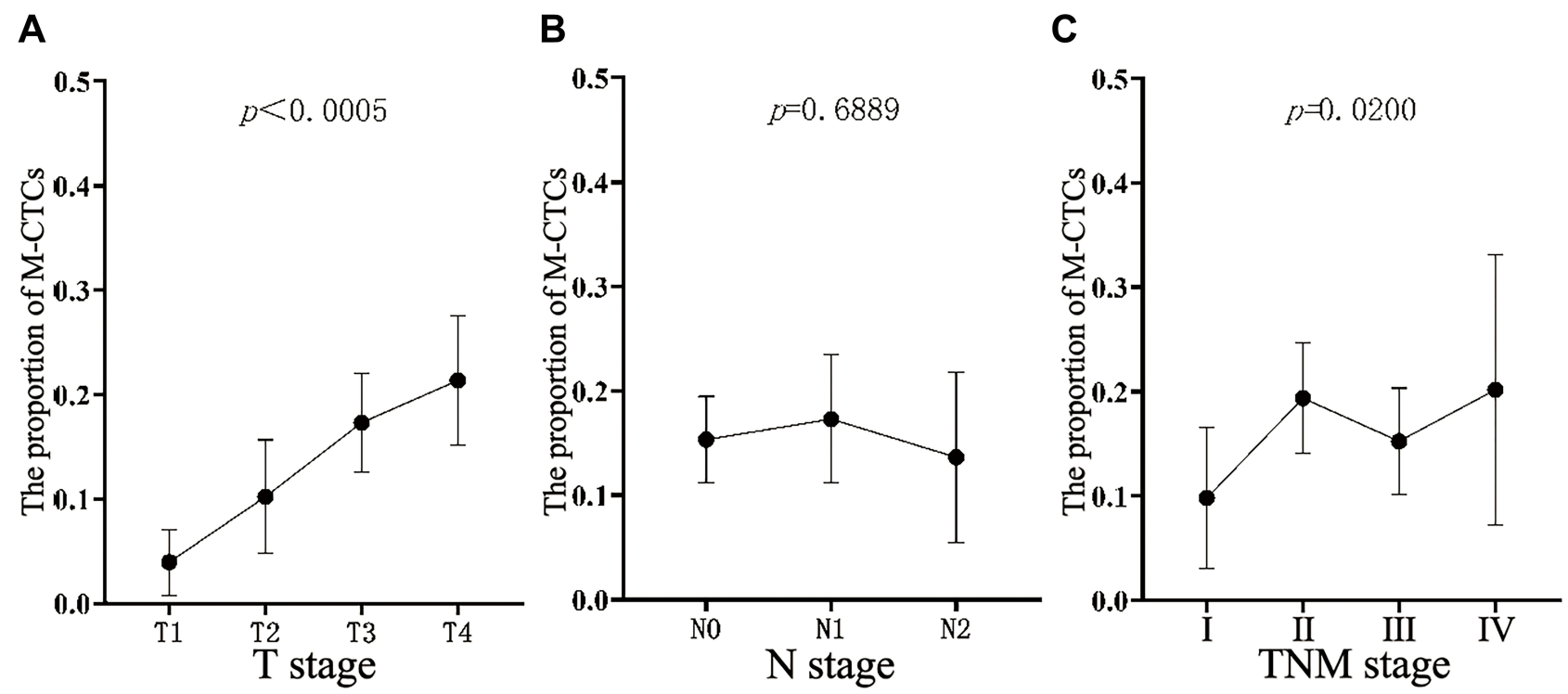

Figure 2 The proportion of M-CTCs in all CTCs. (A) Relationship between the proportion and T stage of pathology; (B) relationship between the proportion and N stage of pathology, and $(\mathbf{C})$ relationship between the proportion and TNM stage of pathology.

Abbreviations: M-CTCs, mesenchymal circulating tumor cells; TNM, tumor- Node-Metastasis.

increased with the continuous progression of the T stage ( $\mathrm{P}$ $<0.0005$ ). Similarly, the TNM stage was also significantly and positively correlated with M-CTCs $(\mathrm{P}<0.0005)$. We found that M-CTCs positive patients had a higher probability of vascular invasion $(80 \%, \mathrm{P}=0.023)$.

Since M-CTCs are significant in the process of cancer development and metastasis, they are considered to be the key to cancer metastasis. On analyzing the relationship between M-CTCs and the pathological T stage and TNM stage, as shown in Figure 2, the proportion of M-CTCs increased continuously with an increasing depth of tumor invasion, and it exhibited a statistically significant difference $(\mathrm{P}<0.0005)$. Similarly, progression of the TNM stage was also statistically associated with the proportion of M-CTCs $(\mathrm{P}=0.02)$. However, no obvious statistical relationship was found in exploring the relationship between the $\mathrm{N}$ stage and M-CTCs $(\mathrm{P}=0.6889)$.

Since M-CTCs play a central role in the development of cancer and are the key to metastasis, we further verified the relationship between the proportion of M-CTCs and $\mathrm{T}$ stage, $\mathrm{N}$ stage, and TNM stage. It can be seen in Figure 2 that both the T stage and TNM stage showed an increasing trend with an increase in the proportion of M-CTCs, but not the $\mathrm{N}$ stage. There was a significant correlation between the T stage and TNM stage and the proportion of M-CTCs $(\mathrm{P}<0.0005$ and $\mathrm{P}=0.0200)$. However, no statistical relationship was found between the $\mathrm{N}$ stage and the proportion of M-CTCs $(\mathrm{p}=0.6889)$.
To further verify the effect of surgery on CTCs, the changes in CTCs numbers at baseline and after surgery were compared; and as shown in Figure 3, a noteworthy decrease in each subpopulation of CTCs was observed. Most patients showed a noticeable decline in CTCs, with a small proportion of patients showing a postoperative increase in CTCs, but an overall decreasing trend was observed. CTCs were dominated by E/M-CTCs, with a low number of E-CTCs and M-CTCs. Concurrently, it was also found that the proportion of M-CTCs returned to zero after surgery in some patients.

Since we hold the notion that the proportion of M-CTCs may affect survival, we compared the proportion of each CTCs subgroup before and after surgery. As seen in Figure 4, the proportions of E-CTCs and E/M-CTCs were barely changed after surgery; however, the proportion of M-CTCs was changed from the statistical perspective after surgical operation.

Our work includes a long-term follow-up of patients, and we could perform Kaplan-Meier analysis for comparing overall survival (OS) and disease-free survival (DFS) of different patients. Although the current follow-up period was not long enough, Figure 5 shows that M-CTCs positive patients at baseline had a significantly worse DFS.

\section{Discussion}

In the present study, we identified CTCs classification with EMT markers in CRC and evaluated the clinical significance of these EMT subtypes. We found that EMT CTCs 
A Totle-CTCs

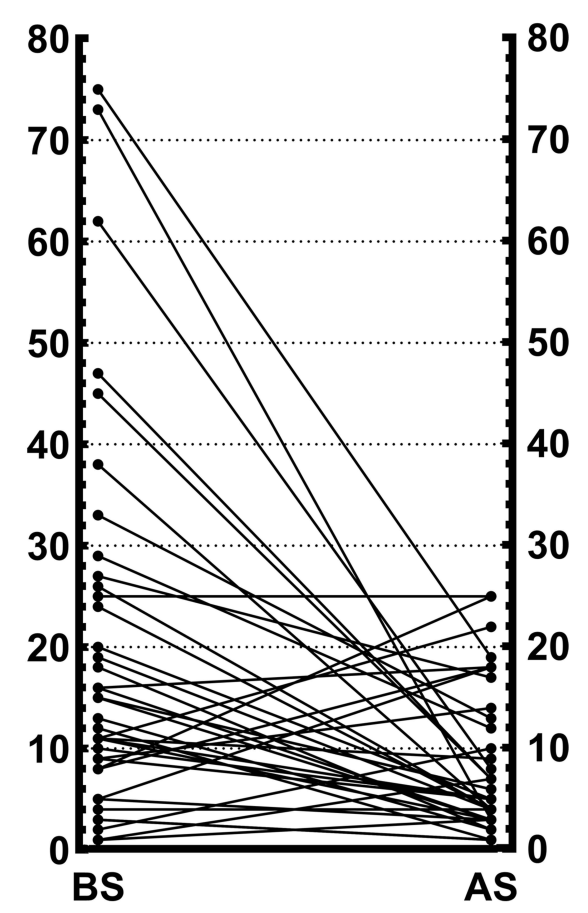

C E/M-CTCs

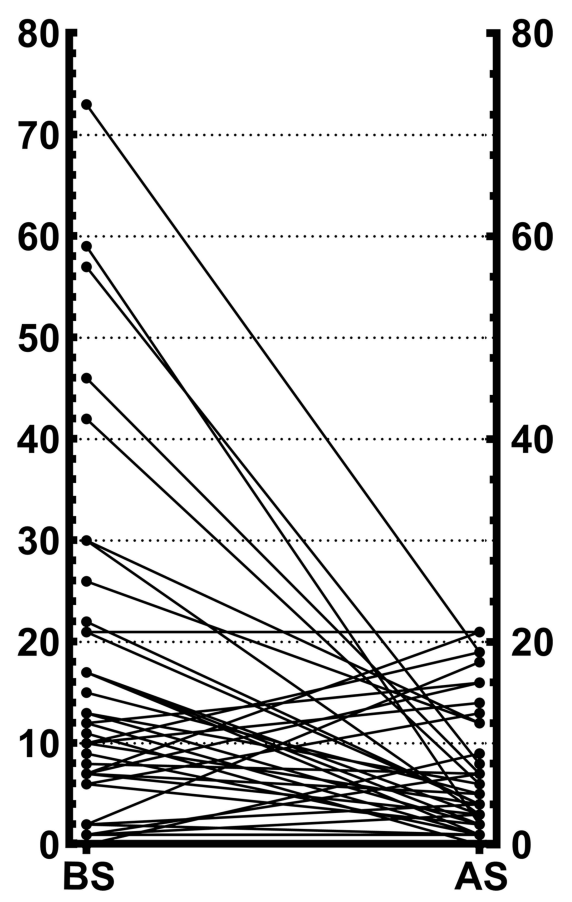

B E-CTCs

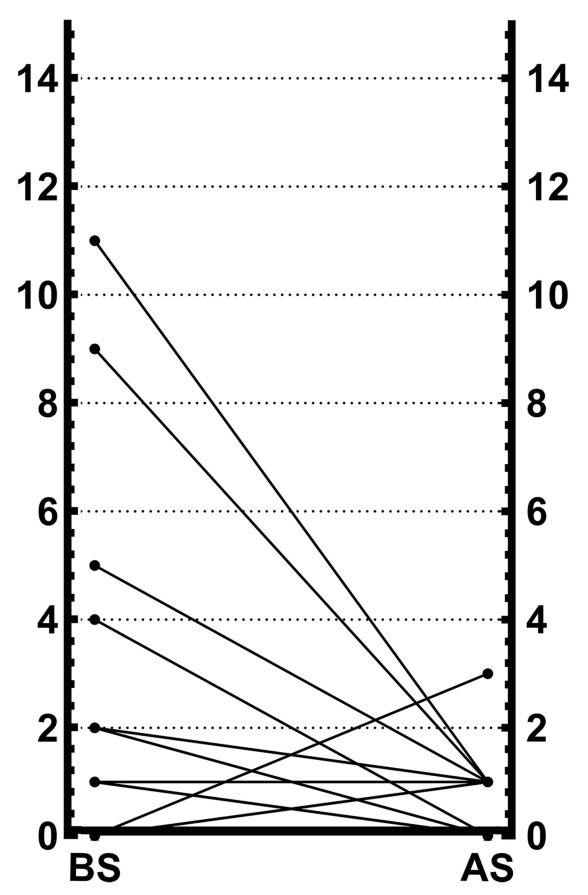

D M-CTCs

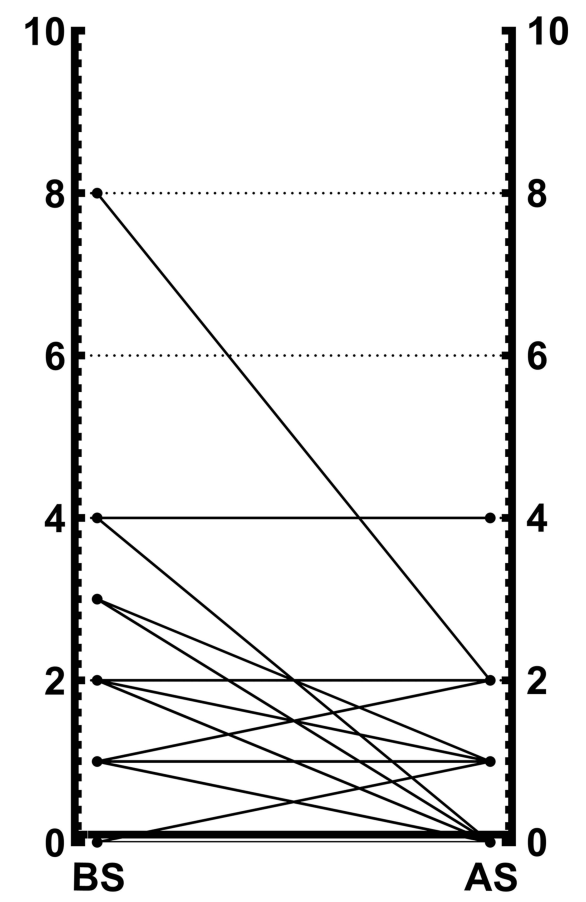

Figure 3 Each phenotype of CTCs before and after surgical treatment. (A) Changes in the total number of CTCs after operation. (B) Changes in the number of E-CTCs after operation. (C) Changes in the number of E/M-CTCs after operation. (D) Changes in the number of M-CTCs after operation.

Abbreviations: BS, before surgery; AS, after surgery; CTCs, circulating tumor cells; E-CTCs, epithelial circulating tumor cells; M-CTCs, mesenchymal circulating tumor cells; E/M-CTCs, epithelial/mesenchymal circulating tumor cells. 


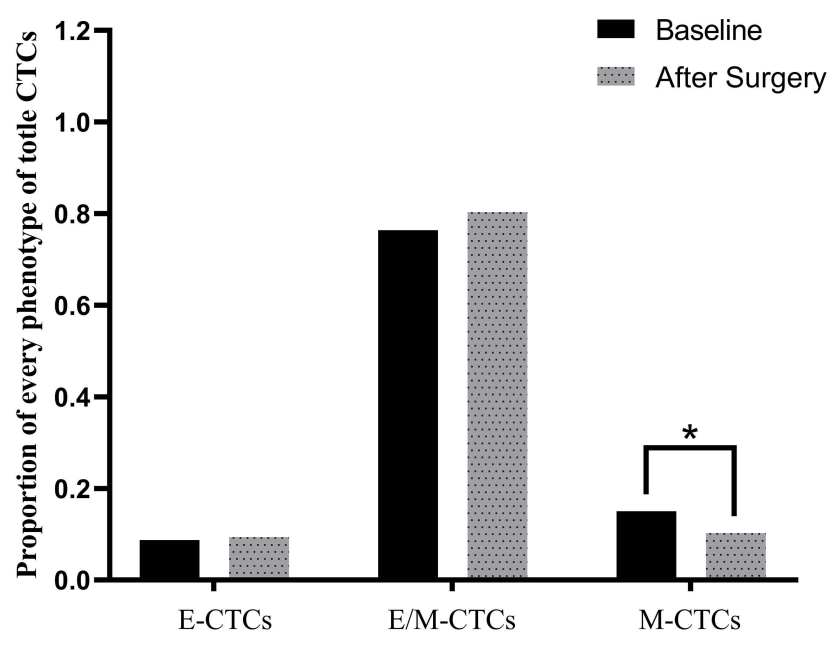

Figure 4 Changes in the proportion of different phenotypes of CTCs before and after operation.

Abbreviation: CTCs, circulating tumor cells.

could add value to the traditional parameters in predicting the postoperative pathological stage, indicating their valuable role in CRC. We isolated CTCs from patients with CRC using the cell size- and phenotype-based CanPatrol technique, which has been reported to detect CTCs with high efficiency. ${ }^{18}$ Using this method, we detected CTCs with different EMT phenotypes in 72 out of the 74 (97.3\%) patients with CRC.

CTCs are closely associated with distant metastasis in various malignancies. M-CTCs are regarded as the most malignant CTCs. ${ }^{19}$ Therefore, M-CTCs positive patients have a greater chance of early recurrence. In addition, we validated using ROC curves, demonstrating that M-CTCs are more suitable as reference indicators (Supplement Figure 1). In this study, we defined M-CTCs $>1 / 7.5 \mathrm{~mL}$ peripheral blood as positive. Firstly, few articles define M-CTCs positive; consequently, there is no definite threshold; Secondly, 1 is the median value of M-CTCs in all patients, which can be used as a cut-off value for segmentation; finally, we used ROC for validation and concluded that using an AUC value of $>1$ as a threshold is superior to using $>0$. We found that the M-CTCs proportion increased with tumor progression; it was especially associated with various pathological parameters, notably, the positive rate of M-CTCs in patients with TNM and T stage IV CRC was $100 \%$ each, and there was a significant association between the proportion of M-CTCs and T stage and TNM stage. These results support the notion of the role of EMT in tumor metastasis, and they indicated that the role of M-CTCs might be more important than that of the other subpopulations in terms of the risk of disease progression. Unlike previous studies, this study did not identify an obvious relationship between the number of CTCs and clinicopathological characteristics except CEA. ${ }^{20,21}$ This may be related to the technique of CTCs isolation and the method of statistical calculation. However, we found that previous studies have mostly used the CellSearch system approved by the FDA for CTCs capture, and the CanPatrol technology used in this study captures a larger variety of CTCs. ${ }^{22,23}$ In addition, when performing the statistical analysis, the total number of CTCs was not grouped, but instead, a $t$-test or nonparametric test was used. However, we found that M-CTCs were associated

\section{B DFS}

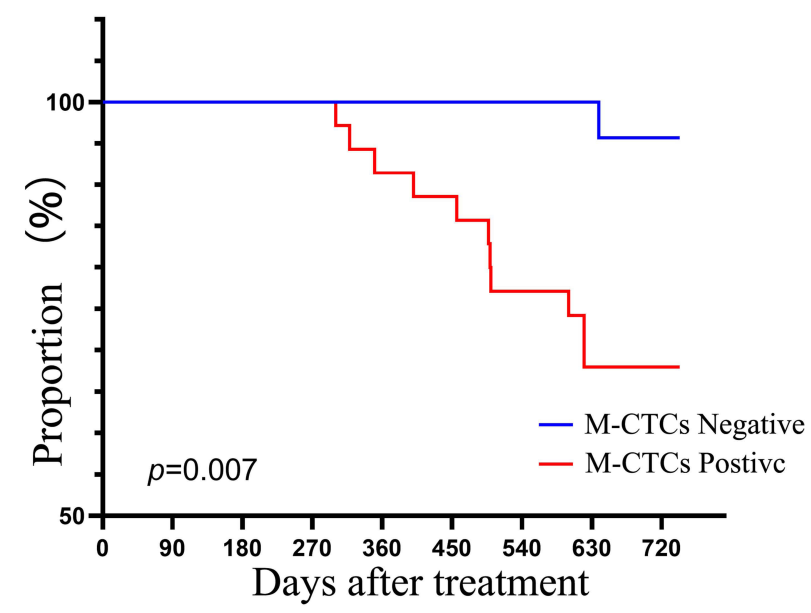

Figure 5 Correlation of positive M-CTCs at baseline with (A) OS and (B) DFS in colorectal cancer patients. Abbreviations: M-CTCs, mesenchymal circulating tumor cells; OS, overall survival; DFS, disease free survival. 
with many clinicopathological parameters, in this study, M-CTCs positive is defined as $>1$, the main reason is that there is no uniform standard at present, and in the subsequent verification of results, it is considered that the critical line of M-CTCs is more valuable (Supplement Figure 2).

Surgery is the most effective way to cure CRC. ${ }^{24,25}$ It is generally believed that the number of CTCs will decrease significantly after surgery. ${ }^{26-28}$ We also verified the postoperative changes in CTCs. However, none of the studies have compared the changes in the proportion of each subtype of CTCs before and after surgery. In this study, all patients who received surgical treatment underwent laparoscopic resection and natural orifice specimen extraction surgery without open surgery. Both procedures are performed laparoscopically, except that the specimen is obtained in a different manner, and the two procedures are considered to have the same therapeutic effect. ${ }^{29,30}$ We first verified that not only the number of CTCs will decrease, but also the proportion of M-CTCs will decrease significantly after surgery, which proves that surgery can reduce the proportion of M-CTCs.

Finally, follow-up was performed, and it revealed that, although it currently appears that there is no significant difference in OS between M-CTCs negative and positive patients, a great difference in DFS has been demonstrated. Because the follow-up period was not long enough, it can be speculated that differences will also occur in future OS. Therefore, high mesenchymal CTCs numbers in CRC patients are indicative of metastasis and recurrence. Our findings further support the notion that M-CTCs are the potential seeds of metastatic dissemination and highlight the potential clinical value of assessing EMT markers in CTCs for the early detection of metastatic tumors. Moreover, we have not only focused on the number of CTCs, but also on the proportion of M-CTCs, which is also associated with tumor prognosis.

Nevertheless, there are some limitations to the present study. They include small sample size and insufficient follow-up time. The number of patients in our study was small and the results should be interpreted with caution, the follow-up period was a maximum of only two years, and a longer follow-up duration should be incorporated with a focus on the patient's condition. Dynamic and multiple monitoring of CTCs is of great significance. Although only one detection of CTCs has prognostic significance, multiple and regular reviews may be more noteworthy. Larger scale, longer time, multicenter clinical trials with more monitoring times are needed to further verify our conclusion and to improve the predictive accuracy.

\section{Conclusion}

Our results indicate that the M-CTCs subpopulation might play an important role in tumor metastasis and is correlated with disease recurrence. In addition, surgery, the most primary treatment for $\mathrm{CRC}$, can effectively reduce not only the number of CTCs but also the proportion of M-CTCs.

\section{Acknowledgments}

This research was supported by the [National Natural Science Foundation of China Grant number: 81872034], [Natural Science Foundation of Heilongjiang Province of China Grant number: H2017016], and [Wu Jieping Medical Foundation Grant number: 320.6750.15183].

\section{Disclosure}

The authors report no conflicts of interest in this work.

\section{References}

1. Bray F, Ferlay J, Soerjomataram I, Siegel RL, Torre LA, Jemal A. Erratum: global cancer statistics 2018: GLOBOCAN estimates of incidence and mortality worldwide for 36 cancers in 185 countries. CA Cancer J Clin. 2020;70(4):313. doi:10.3322/caac.21609

2. Yeh CC, Hsi SC, Chuu CP, Kao YH. Synchronous triple carcinoma of the colon and rectum. World J Surg Oncol. 2013;11:66. doi:10.1186/ 1477-7819-11-66

3. Nicolazzo C, Raimondi C, Gradilone A, et al. Circulating tumor cells in right- and left-sided colorectal cancer. Cancers (Basel). 2019;11 (8). doi:10.3390/cancers11081042

4. Masuda T, Hayashi N, Iguchi T, Ito S, Eguchi H, Mimori K. Clinical and biological significance of circulating tumor cells in cancer. $\mathrm{Mol}$ Oncol. 2016;10(3):408-417. doi:10.1016/j.molonc.2016.01.010

5 . Kemeny N. The management of resectable and unresectable liver metastases from colorectal cancer. Curr Opin Oncol. 2010;22 (4):364-373. doi:10.1097/CCO.0b013e32833a6c8a

6. Hartkopf AD, Wagner P, Wallwiener D, Fehm T, Rothmund R. Changing levels of circulating tumor cells in monitoring chemotherapy response in patients with metastatic breast cancer. Anticancer Res. 2011;31(3):979-984.

7. Smerage JB, Barlow WE, Hortobagyi GN, et al. Circulating tumor cells and response to chemotherapy in metastatic breast cancer: SWOG S0500. J Clin Oncol. 2014;32(31):3483-3489. doi:10.1200/ JCO.2014.56.2561

8. Hardingham JE, Grover P, Winter M, Hewett PJ, Price TJ, Thierry B. Detection and clinical significance of circulating tumor cells in colorectal cancer - 20 years of progress. Mol Med. 2015;21(Suppl1):S2531. doi:10.2119/molmed.2015.00149

9. Tol J, Koopman M, Miller MC, et al. Circulating tumour cells early predict progression-free and overall survival in advanced colorectal cancer patients treated with chemotherapy and targeted agents. Ann Oncol. 2010;21(5):1006-1012. doi:10.1093/annonc/mdp463

10. Wu F, Zhu J, Mao Y, Li X, Hu B, Zhang D. Associations between the epithelial-mesenchymal transition phenotypes of circulating tumor cells and the clinicopathological features of patients with colorectal cancer. Dis Markers. 2017;2017:9474532. doi:10.1155/2017/9474532 
11. Werner S, Stenzl A, Pantel K, Todenhöfer T. Expression of epithelial mesenchymal transition and cancer stem cell markers in circulating tumor cells. Adv Exp Med Biol. 2017;994:205-228.

12. Satelli A, Mitra A, Brownlee Z, et al. Epithelial-mesenchymal transitioned circulating tumor cells capture for detecting tumor progression. Clin Cancer Res. 2015;21(4):899-906. doi:10.1158/ 1078-0432.CCR-14-0894

13. Mooney SM, Talebian V, Jolly MK, et al. The GRHL2/ZEB feedback loop - a key axis in the regulation of EMT in breast cancer. $J$ Cell Biochem. 2017;118(9):2559-2570. doi:10.1002/jcb.25974

14. Li -T-T, Liu H, Li F-P, et al. Evaluation of epithelial-mesenchymal transitioned circulating tumor cells in patients with resectable gastric cancer: relevance to therapy response. World J Gastroenterol. 2015;21(47):13259-13267. doi:10.3748/wjg.v21.i47.13259

15. Hu B, Yang X-R, Xu Y, et al. Systemic immune-inflammation index predicts prognosis of patients after curative resection for hepatocellular carcinoma. Clin Cancer Res. 2014;20(23):6212-6222. doi:10.1158/1078-0432.CCR-14-0442

16. Abdallah EA, Braun AC, Flores B, et al. The potential clinical implications of circulating tumor cells and circulating tumor microemboli in gastric cancer. Oncologist. 2019;24(9):e854-e863. doi:10.1634/theoncologist.2018-0741

17. Wu S, Liu S, Liu Z, et al. Classification of circulating tumor cells by epithelial-mesenchymal transition markers. PLoS One. 2015;10(4): e0123976. doi:10.1371/journal.pone.0123976

18. Qi LN, Xiang BD, Wu FX, et al. Circulating tumor cells undergoing EMT provide a metric for diagnosis and prognosis of patients with hepatocellular carcinoma. Cancer Res. 2018;78(16):4731-4744. doi:10.1158/0008-5472.CAN-17-2459

19. Hou J, Guo C, Lyu G. Clinical significance of epithelial-mesenchymal transition typing of circulating tumour cells in colorectal cancer. Colorectal Dis. 2020;22(5):581-587. doi:10.1111/codi.14927

20. Denève E, Riethdorf $\mathrm{S}$, Ramos $\mathrm{J}$, et al. Capture of viable circulating tumor cells in the liver of colorectal cancer patients. Clin Chem. 2013;59(9):1384-1392. doi:10.1373/clinchem.2013.202846
21. Shimada R, Iinuma $H$, Akahane $T$, Horiuchi $A$, Watanabe $T$. Prognostic significance of CTCs and CSCs of tumor drainage vein blood in Dukes' stage B and C colorectal cancer patients. Oncol Rep. 2012;27(4):947-953. doi:10.3892/or.2012.1649

22. Allard WJ, Matera J, Miller MC, et al. Tumor cells circulate in the peripheral blood of all major carcinomas but not in healthy subjects or patients with nonmalignant diseases. Clin Cancer Res. 2004;10 (20):6897-6904. doi:10.1158/1078-0432.CCR-04-0378

23. van Dalum G, Stam GJ, Scholten LF, et al. Importance of circulating tumor cells in newly diagnosed colorectal cancer. Int J Oncol. 2015;46(3):1361-1368. doi:10.3892/ijo.2015.2824

24. Brenner H, Kloor M, Pox CP. Colorectal cancer. Lancet. 2014;383 (9927):1490-1502. doi:10.1016/S0140-6736(13)61649-9

25. Gu J, Chen N. Current status of rectal cancer treatment in China. Colorectal Dis. 2013;15(11):1345-1350. doi:10.1111/codi.12269

26. Li W, Zhou X, Huang Z, et al. Laparoscopic surgery minimizes the release of circulating tumor cells compared to open surgery for hepatocellular carcinoma. Surg Endosc. 2015;29(11):3146-3153. doi:10.1007/s00464-014-4041-5

27. Yin W, Han YM, Li ZL, Huang ZX, Huang L, Zhong XG. Clinical significance of perioperative EMT-CTC in rectal cancer patients receiving open/laparoscopic surgery. Neoplasma. 2020;67 (5):1131-1138. doi:10.4149/neo_2020_190709N611

28. Wu CY, Lee CL, Wu CF, et al. Circulating tumor cells as a tool of minimal residual disease can predict lung cancer recurrence: a longitudinal, prospective trial. Diagnostics (Basel). 2020;10(3):144.

29. Zhong Y, Qiao T, Ma T, et al. Long-term outcome comparison between two specimen extraction approaches for middle rectum cancer: a Retrospective Study. Surg Innov. Epub 2021 Mar 31; doi:10.1177/15533506211006970

30. Tang Q, Zhu Y, Xiong H, et al. Natural orifice specimen extraction surgery versus conventional laparoscopic-assisted resection in the treatment of colorectal cancer: a propensity-score matching study. Cancer Manag Res. 2021;13:2247-2257. doi:10.2147/CMAR. S291085

\section{Publish your work in this journal}

Cancer Management and Research is an international, peer-reviewed open access journal focusing on cancer research and the optimal use of preventative and integrated treatment interventions to achieve improved outcomes, enhanced survival and quality of life for the cancer patient.
The manuscript management system is completely online and includes a very quick and fair peer-review system, which is all easy to use. Visit http://www.dovepress.com/testimonials.php to read real quotes from published authors. 